\title{
Survey of EMF Emitted from Lab Equipments and Air Conditioners in Textile Engineering Labs of Southeast University in Bangladesh
}

\author{
Munima Haque $^{1}$, Md. Quamruzzaman ${ }^{2}$ \\ Assistant Professor, Department of Electrical and Electronic Engineering, Southeast University, Dhaka, Bangladesh ${ }^{1}$ \\ Associate Professor, Department of Electrical and Electronic Engineering, Southeast University, Dhaka, Bangladesh ${ }^{2}$
}

\begin{abstract}
The aim of this survey is to investigate whether the Electromagnetic Fields (EMF) emitted from various lab equipments affects the students and employees. There is a standard threshold value recommended by WHO for both electric and magnetic fields. Electro-Magnetic Fields is emitted from high power transmission lines, computer monitor/video display unit, radio waves of different frequencies (extremely low frequency to microwaves), telecommunication, satellite, radar etc. which causes health hazards to living system and environment. There has not been much study performed in Bangladesh. The data were collected from various lab equipments and air conditioners of different textile engineering labs at Southeast University in Dhaka, Bangladesh. Both threshold values of Electric and Magnetic fields were measured for various electronic equipments. Also the maximum value of the magnetic field results showed that in many cases the magnetic field radiated from the different sources are greater than the threshold limit which are the main point of our findings.
\end{abstract}

Keywords: EMF, NIR, WHO, ELF, EF, MF

\section{INTRODUCTION}

Ionizing radiation is the radiation of sufficiently high above the naturally occurring ambient electric and energy to cause ionization in the medium through which it magnetic fields of $10^{-4} \mathrm{~V} / \mathrm{m}$ and $10^{-13} \mathrm{~T}$ respectively [1-2]. passes. It may consist of a stream of high-energy particle Lower animals are reported to very much sensitive to (e.g. electron, protons, alpha particles) or short wavelength electromagnetic fields. It is observed that animals like rats electromagnetic radiation (ultraviolet, X-rays, gamma- make their living brooding holes away from the high rays). Radiation, which does not cause any ionization of electric field and bees block-up their hives in the chronic the media while passing through it, is known a non- presence of NIR/EMF. As a consequence, scientists and ionizing radiation (NIR). Examples of non-ionizing health physicians in developed countries have become radiation are ultraviolet, visible light, infrared, microwave aware of the effects of NIR [3]. Their research and and radiowave. Their energy is relatively low; it only observations have brought out some remarkable results manages to cause molecules to vibrate and induces heating effects.

Exposure to Extremely Low Frequency (ELF) electric and magnetic fields does produce biological effects. However, except for fields strong enough to induce current densities above the threshold for the stimulation of nerve tissue, there is no consensus as to whether these effects constitute a hazard to human health. Human data from epidemiological studies, including reported effects on cancer promotion, congenital malformations, reproductive performance and general health, though somewhat suggestive to adverse health effects, are not conclusive.

Since magnetic field is more harmful than electric field, there must be a limit both in the residential and occupational levels between 0.2 to $0.3 \mu \mathrm{T}$ or $2.5 \mathrm{mG}$. (This value is internationally recognized as standard limit in many countries). Also, for electric field this value is $25 \mathrm{~V} / \mathrm{m}$. It must be ensured that intensity of radiation in the body does not exceed the recommended maximum level $\left(10 \mathrm{~mW} / \mathrm{cm}^{2}, 195 \mathrm{~V} / \mathrm{m}\right.$ in U.S.A. and $0.1 \mathrm{~mW} / \mathrm{cm}^{2}, 20 \mathrm{~V} / \mathrm{m}$ in CIS). these human made electric and magnetic fields (typically $25 \mathrm{~V} / \mathrm{m}$ and $2.5 \mathrm{mG}$ or $0.25 \mu \mathrm{T}$ ) are substantially

linking low level alternating electromagnetic fields with serious health hazards. There is also evidence that biological effect like immune deficiency, sensitive lymphocytes, disrupting DNA, cellular breakdown is being affected by NIR.

Some research has been performed in this regard. Most recently, Epidemiological survey of people working in EMF field exposed to high frequency have been investigated [5]. Also, research was performed for epidemiological survey on effect of EMF emitted by photocopy machines generally used in Dhaka city Bangladesh [6]. Survey was done on EMF emitted by Lab equipments in various labs of Southeast University in Bangladesh for possible health hazards [7]. A case study was done on EMF near high voltage transmission line [8]. Also, a review was done on Non Ionizing Radiation (NIR), its harmful effects especially from Mobile/Cell Phone and Towers [9].

There have been various papers published on EMF of radio, TV etc. but not much on air conditioners. Since the invention of modern electrical air conditioning unit in 1902 by Willis Carrier, Buffalo, New York (4) there has 
been tremendous increase in use of air conditioning in the threshold values and also if the students and employees are world. In some cases it has been used in cooling the safe from these equipments. building, theatres, and for commercial purposes. Since air conditioner has been used for comfort, its demand has increased. After the invention of portable air conditioners, it has been easier to purchase one. Especially in tropical countries like Bangladesh, it is in high demand not only at offices, but at homes also. With the increase in the efficiency of the modern air conditioners as well as the attractive decrease in its price, offices and private homes have their own air conditioner systems. At Southeast University, all the offices, labs and classrooms have air conditioners due to very hot and humid weather during the summer. Each office, labs and classrooms have one or multiple switchboards. The students spend around 7-8 hours each day in these classrooms and labs which the faculties and employees also do the same. The aim of this research is to investigate whether the EMF emitted from these air conditioners and switchboards are within

\section{METHODS}

A magnetic Science International MF meter (Serial No 624335) was used for measuring the magnetic field values for the various air conditioners and switch board equipments. A Coghill Field Mouse for Biohazard Awareness was used for measuring the threshold values for both electric field (EF) and magnetic field (MF) around the instrument. The readings were taken to cover all around the equipment.

\section{III.RESULTS}

Findings at different EMF sources: All the readings were taken from different labs of Textile Engineering department, Southeast University Tejgaon Permanent campus. Readings were taken from lab equipments of each lab.

Table 1: EMF values measured for lab equipments in various Textile Engineering labs in Southeast University (SEU), November 2015. Location address: (1) EE lab, (2) Textile Testing and Quality Control lab, (3) Workshop practice lab, (4) Yarn manufacturing technology lab, (5) Physics lab.

\begin{tabular}{|c|c|c|c|c|c|c|c|c|c|c|c|}
\hline \multirow[t]{2}{*}{$\begin{array}{l}\text { Serial } \\
\text { No. }\end{array}$} & \multirow[t]{2}{*}{$\begin{array}{l}\text { Equipment info. } \\
\text { (Machine \#, } \\
\text { Machine Model, } \\
\text { made country, } \\
\text { year made, Date of } \\
\text { installation) }\end{array}$} & \multicolumn{2}{|c|}{$\begin{array}{l}\text { Threshold dis. in } \\
\text { front of the } \\
\text { equipment } \\
\text { measured from } \\
\text { the centre of the } \\
\text { equipment }(\mathrm{cm})\end{array}$} & \multirow[t]{2}{*}{$\begin{array}{l}\text { Magnetic } \\
\text { Field } \\
\text { (mG) in } \\
\text { front of } \\
\text { the eqpt. }\end{array}$} & \multicolumn{2}{|c|}{$\begin{array}{l}\text { Threshold } \\
\text { dis. at right } \\
\text { side of the } \\
\text { equipment } \\
\text { (cm) }\end{array}$} & \multirow[t]{2}{*}{$\begin{array}{l}\text { Magnetic } \\
\text { Field (mG) } \\
\text { at right side } \\
\text { of the } \\
\text { equipment }\end{array}$} & \multicolumn{2}{|c|}{$\begin{array}{l}\text { Threshold } \\
\text { distance at the } \\
\text { left side of the } \\
\text { equipment } \\
\text { (cm) }\end{array}$} & \multirow[t]{2}{*}{$\begin{array}{l}\text { Magnetic } \\
\text { Field } \\
(\mathrm{mG}) \text { at } \\
\text { left side } \\
\text { of the } \\
\text { equipment }\end{array}$} & \multirow[t]{2}{*}{$\begin{array}{l}\text { Magnetic } \\
\text { Field } \\
\text { maximum } \\
(\mathrm{mG})\end{array}$} \\
\hline & & EF & $\mathrm{MF}$ & & EF & $\mathrm{MF}$ & & EF & MF & & \\
\hline $1 \mathrm{a}$ & $\begin{array}{l}\text { YOKOGAYAWA } \\
\text { AL310.35 Hz } \\
\text { oscilloscope } \\
\text { Model- } 366531 \\
\text { China 06.05.2010 }\end{array}$ & $6.5 \mathrm{~cm}$ & * & $8 \mathrm{mG}$ & $\begin{array}{l}4.5 \\
\mathrm{~cm}\end{array}$ & $*$ & $18 \mathrm{mG}$ & $4.5 \mathrm{~cm}$ & $*$ & $8 \mathrm{mG}$ & $18 \mathrm{mG}$ \\
\hline $1 b$ & $\begin{array}{l}\text { YOKOGAYAWA } \\
\text { Fe } 35 . \mathrm{Hz} \\
\text { oscilloscope } \\
\text { Model- } 366531 \\
\text { Taiwan } 06.05 .2010\end{array}$ & $20 \mathrm{~cm}$ & $*$ & $11 \mathrm{mG}$ & $26 \mathrm{~cm}$ & $*$ & $28 \mathrm{mG}$ & $20 \mathrm{~cm}$ & $*$ & $13 \mathrm{mG}$ & $28 \mathrm{mG}$ \\
\hline $1 \mathrm{c}$ & $\begin{array}{l}\text { YOKOGAYAWA } \\
\text { Fe } 35 . \mathrm{Hz} \\
\text { oscilloscope } \\
\text { Model- } 366531 \\
\text { Taiwan06.05.2010 }\end{array}$ & $14 \mathrm{~cm}$ & $*$ & $5 \mathrm{mG}$ & $7 \mathrm{~cm}$ & $*$ & $4 \mathrm{mG}$ & $14.5 \mathrm{~cm}$ & $*$ & $18 \mathrm{mG}$ & $18 \mathrm{mG}$ \\
\hline $1 d$ & $\begin{array}{l}\text { McP. DC power } \\
\text { supply } \\
\text { Model-M10- } \\
\text { SP30032 } \\
\text { China, 6-05-2010 }\end{array}$ & $17 \mathrm{~cm}$ & $*$ & $67 \mathrm{mG}$ & $18 \mathrm{~cm}$ & $*$ & $114 \mathrm{mG}$ & $17 \mathrm{~cm}$ & $*$ & $94 \mathrm{mG}$ & $114 \mathrm{mG}$ \\
\hline $1 \mathrm{e}$ & $\begin{array}{l}\text { Function generator } \\
\text { Model: FG-35 } \\
\text { China In. date: } \\
6.5 .2015\end{array}$ & $*$ & $*$ & $16 \mathrm{mG}$ & $2 \mathrm{~cm}$ & $*$ & $12 \mathrm{mG}$ & $6 \mathrm{~cm}$ & $*$ & $25 \mathrm{mG}$ & $25 \mathrm{mG}$ \\
\hline $1 \mathrm{f}$ & $\begin{array}{l}\text { Oscilloscope } \\
\text { AL310, } \\
\text { model:366531, } \\
\text { China, Ins. Date: } \\
\text { 6-5-2015 }\end{array}$ & $*$ & * & $4 \mathrm{mG}$ & $8 \mathrm{~cm}$ & * & $22 \mathrm{mG}$ & $0.5 \mathrm{~cm}$ & $*$ & $11 \mathrm{mG}$ & $22 \mathrm{mG}$ \\
\hline $1 \mathrm{~g}$ & $\begin{array}{l}\text { McP. DC power } \\
\text { supply } \\
\text { Model-M10- } \\
\text { SP3003,L } \\
\text { China, 6-05-2010 }\end{array}$ & $67 \mathrm{~cm}$ & $*$ & $68 \mathrm{mG}$ & $64 \mathrm{~cm}$ & $*$ & $94 \mathrm{mG}$ & $162 \mathrm{~cm}$ & $*$ & $108 \mathrm{mG}$ & $108 \mathrm{mG}$ \\
\hline $1 \mathrm{~h}$ & $\begin{array}{l}\text { McP. DC power } \\
\text { supply } \\
\text { Model-FG-35 } \\
\text { China, 6-05-2015 }\end{array}$ & $*$ & $*$ & $16 \mathrm{mG}$ & $\begin{array}{l}2.3 \\
\mathrm{~cm}\end{array}$ & $*$ & $12 \mathrm{mG}$ & $5.5 \mathrm{~cm}$ & $*$ & $24 \mathrm{mG}$ & $24 \mathrm{mG}$ \\
\hline
\end{tabular}


INTERNATIONAL JOURNAL OF INNOVATIVE RESEARCH IN ELECTRICAL, ELECTRONICS, INSTRUMENTATION AND CONTROL ENGINEERING Vol. 4, Issue 3, March 2016

\begin{tabular}{|c|c|c|c|c|c|c|c|c|c|c|c|}
\hline $2 a$ & $\begin{array}{l}\text { Pendulam, model: } \\
\text { MAG 40211, } \\
\text { India, D.I.: 27-08- } \\
\text { 2008, Serial no. } \\
\text { 189011, } \\
\text { capacity:300 lbs. }\end{array}$ & $\begin{array}{l}17.5 \\
\mathrm{~cm}\end{array}$ & $*$ & $150 \mathrm{mG}$ & $6 \mathrm{~cm}$ & $*$ & $9 \mathrm{mG}$ & $2 \mathrm{~cm}$ & $*$ & $10 \mathrm{mG}$ & $150 \mathrm{mG}$ \\
\hline $2 b$ & $\begin{array}{l}\text { Pilling and } \\
\text { Snagging Tester, } \\
\text { model: GT-C18A, } \\
\text { China, D.I.: } 23-3- \\
2015\end{array}$ & $45 \mathrm{~cm}$ & $*$ & $9 \mathrm{mG}$ & $53 \mathrm{~cm}$ & $*$ & $17 \mathrm{mG}$ & $53 \mathrm{~cm}$ & $*$ & $10 \mathrm{mG}$ & $17 \mathrm{mG}$ \\
\hline $2 \mathrm{c}$ & $\begin{array}{l}\text { Electric Balance, } \\
\text { model: FX-300i, } \\
\text { Japan, D.I.: 22-03- } \\
2015\end{array}$ & $76 \mathrm{~cm}$ & $*$ & $9 \mathrm{mG}$ & $27 \mathrm{~cm}$ & $*$ & $4 \mathrm{mG}$ & $102 \mathrm{~cm}$ & $*$ & $7 \mathrm{mG}$ & $9 \mathrm{mG}$ \\
\hline $2 d$ & $\begin{array}{l}\text { Dry oven, 22-09- } \\
\text { 2015, Memment, } \\
\text { Model: } 30-1060\end{array}$ & $35 \mathrm{~cm}$ & $*$ & $12 \mathrm{mG}$ & $21 \mathrm{~cm}$ & $*$ & $5 \mathrm{mG}$ & $15 \mathrm{~cm}$ & $*$ & $9 \mathrm{mG}$ & $12 \mathrm{mG}$ \\
\hline $2 \mathrm{e}$ & $\begin{array}{l}\text { Air compressor, } \\
\text { Model: HD } 0208\end{array}$ & $52 \mathrm{~cm}$ & $*$ & $600 \mathrm{mG}$ & $36 \mathrm{~cm}$ & * & $65 \mathrm{mG}$ & $27 \mathrm{~cm}$ & $*$ & $103 \mathrm{mG}$ & $600 \mathrm{mG}$ \\
\hline $3 a$ & $\begin{array}{l}\text { Drilling machine } \\
\text { Type: LT-16J, } \\
\text { capacity: } 16 \mathrm{~mm} \text {, } \\
\text { Voltage: } 220 \mathrm{~V}, \\
\text { Power: } 1 / 2 \mathrm{Hp} \text {, No. } \\
\text { 20110830, China }\end{array}$ & $17 \mathrm{~cm}$ & $*$ & $13 \mathrm{mG}$ & $57 \mathrm{~cm}$ & $*$ & $199 \mathrm{mG}$ & $34 \mathrm{~cm}$ & $*$ & $231 \mathrm{mG}$ & $231 \mathrm{mG}$ \\
\hline $3 b$ & $\begin{array}{l}\text { Air compressor } \\
\text { Model: V-0.03, } \\
\text { China, July } 2012 \\
03.07 .2013\end{array}$ & $20 \mathrm{~cm}$ & $*$ & $320 \mathrm{mG}$ & $42 \mathrm{~cm}$ & $*$ & $600 \mathrm{mG}$ & $67 \mathrm{~cm}$ & $*$ & $590 \mathrm{mG}$ & $600 \mathrm{mG}$ \\
\hline $5 a$ & $\begin{array}{l}\text { Digital power } \\
\text { supply } \\
\text { PS-303 TD, } \\
110 / 230 \mathrm{~V}, \\
\text { Taiwan, Ins. Date: } \\
24-10-11\end{array}$ & $20 \mathrm{~cm}$ & $*$ & $32 \mathrm{mG}$ & $30 \mathrm{~cm}$ & & $62 \mathrm{mG}$ & $39 \mathrm{~cm}$ & & $93 \mathrm{mG}$ & $93 \mathrm{mG}$ \\
\hline $5 b$ & $\begin{array}{l}\text { Digital power } \\
\text { supply } \\
\text { PS-153 TD, 15V, } \\
\text { Taiwan, Ins. Date: } \\
24-10-11\end{array}$ & $31 \mathrm{~cm}$ & $*$ & $23 \mathrm{mG}$ & $42 \mathrm{~cm}$ & $*$ & $54 \mathrm{mG}$ & $26 \mathrm{~cm}$ & $*$ & $54 \mathrm{mG}$ & $54 \mathrm{mG}$ \\
\hline $5 c$ & $\begin{array}{l}\text { DC power supply } \\
\text { SAKO } \\
\text { SK-1730SC2A, } \\
\text { Germany } \\
8-02-2008\end{array}$ & $28 \mathrm{~cm}$ & $*$ & $13 \mathrm{mG}$ & $26 \mathrm{~cm}$ & $*$ & $47 \mathrm{mG}$ & $25 \mathrm{~cm}$ & $*$ & $31 \mathrm{mG}$ & $47 \mathrm{mG}$ \\
\hline $5 d$ & $\begin{array}{l}\text { DC power supply } \\
\text { SAKO } \\
\text { SK-1730SC2A, } \\
\text { Germany } \\
\text { 10-01-2007 }\end{array}$ & $21 \mathrm{~cm}$ & $*$ & $14 \mathrm{mG}$ & $22 \mathrm{~cm}$ & $*$ & $51 \mathrm{mG}$ & $14 \mathrm{~cm}$ & $*$ & $25 \mathrm{mG}$ & $51 \mathrm{mG}$ \\
\hline $5 e$ & $\begin{array}{l}\text { Power supply for } \\
\text { sodium vapor } \\
\text { lamp, 35 Watts } \\
\text { Bangladesh, 15-6- } \\
2008\end{array}$ & $89 \mathrm{~cm}$ & $*$ & $94 \mathrm{mG}$ & $85 \mathrm{~cm}$ & $*$ & $102 \mathrm{mG}$ & $100 \mathrm{~cm}$ & $*$ & $104 \mathrm{mG}$ & $104 \mathrm{mG}$ \\
\hline $5 \mathrm{f}$ & $\begin{array}{l}\text { Sodium lamp } \\
\text { transformer, } \\
\text { Bioman 35W, 20- } \\
\text { 3-2007 }\end{array}$ & $93 \mathrm{~cm}$ & $*$ & $89 \mathrm{mG}$ & $\begin{array}{l}100 \\
\mathrm{~cm}\end{array}$ & $*$ & $113 \mathrm{mG}$ & $97 \mathrm{~cm}$ & $*$ & $121 \mathrm{mG}$ & $121 \mathrm{mG}$ \\
\hline
\end{tabular}


Table 2: EMF values measured for air conditioners in various Textile Engineering labs in Southeast University (SEU), November 2015. Location address: (1) EE lab, (2) Textile Testing and Quality Control lab, (3) Workshop practice lab, (4) Yarn manufacturing technology lab, (5) Physics lab.

\begin{tabular}{|c|c|c|c|c|c|c|c|c|c|c|c|}
\hline \multirow[t]{2}{*}{$\begin{array}{l}\text { Serial } \\
\text { No. }\end{array}$} & \multirow[t]{2}{*}{$\begin{array}{l}\text { Equipment info. } \\
\text { (Machine \#, } \\
\text { Machine Model, } \\
\text { made country, } \\
\text { year made, Date of } \\
\text { installation) }\end{array}$} & \multicolumn{2}{|c|}{$\begin{array}{l}\text { Threshold dis. in } \\
\text { front of the } \\
\text { equipment } \\
\text { measured from } \\
\text { the centre of the } \\
\text { equipment }(\mathrm{cm})\end{array}$} & \multirow[t]{2}{*}{$\begin{array}{l}\text { Magnet } \\
\text { ic Field } \\
(\mathrm{mG}) \\
\text { in front } \\
\text { of the } \\
\text { eqpt. }\end{array}$} & \multicolumn{2}{|c|}{$\begin{array}{l}\text { Threshold dis. } \\
\text { at right side of } \\
\text { the equipment } \\
(\mathrm{cm})\end{array}$} & \multirow[t]{2}{*}{$\begin{array}{l}\text { Magnetic } \\
\text { Field } \\
(\mathrm{mG}) \text { at } \\
\text { right side } \\
\text { of the } \\
\text { equipment }\end{array}$} & \multicolumn{2}{|c|}{$\begin{array}{l}\text { Threshold } \\
\text { distance at } \\
\text { the left side } \\
\text { of the } \\
\text { equipment } \\
\text { (cm) }\end{array}$} & \multirow[t]{2}{*}{$\begin{array}{l}\text { Magnetic } \\
\text { Field } \\
\text { (mG) at } \\
\text { left side } \\
\text { of the } \\
\text { equipment }\end{array}$} & \multirow[t]{2}{*}{$\begin{array}{l}\text { Magnetic } \\
\text { Field } \\
\text { maximum } \\
(\mathrm{mG})\end{array}$} \\
\hline & & EF & MF & & EF & MF & & $\mathrm{EF}$ & MF & & \\
\hline $1 \mathrm{a}$ & $\begin{array}{l}\text { Power- } 3 \text { ton } \\
\text { Brand: Carrier } \\
\text { Model: } 38 \text { VTA } 040 \\
\text {..90125 Phase-3 } \\
\text { Ins. Date: } 5-5-2014\end{array}$ & $3 \mathrm{~cm}$ & $*$ & $4 \mathrm{mG}$ & $2 \mathrm{~cm}$ & $*$ & $3 \mathrm{mG}$ & $3 \mathrm{~cm}$ & $*$ & $4 \mathrm{mG}$ & $4 \mathrm{mG}$ \\
\hline $1 \mathrm{~b}$ & $\begin{array}{l}\text { Power- } 3 \text { ton } \\
\text { Brand: Carrier } \\
\text { Model: } 38 \text { VTA } 040 \\
\text {...90125 Phase-3 } \\
\text { Ins. Date: } 5-5-2014\end{array}$ & $1 \mathrm{~cm}$ & * & $10 \mathrm{mG}$ & $2 \mathrm{~cm}$ & * & $9 \mathrm{mG}$ & $3 \mathrm{~cm}$ & $*$ & $10 \mathrm{mG}$ & $10 \mathrm{mG}$ \\
\hline $2 a$ & $\begin{array}{l}\text { Power- } 3 \text { ton } \\
\text { Brand: Carrier } \\
\text { Model: } 38 \text { VTA } 040 \\
\text {...90125 Phase-3 } \\
\text { Ins. Date: } 5-5-2014\end{array}$ & $3 \mathrm{~cm}$ & $*$ & $4 \mathrm{mG}$ & $3 \mathrm{~cm}$ & $*$ & $3 \mathrm{mG}$ & $2 \mathrm{~cm}$ & $*$ & $3 \mathrm{mG}$ & $4 \mathrm{mG}$ \\
\hline $2 b$ & $\begin{array}{l}\text { Power- } 3 \text { ton } \\
\text { Brand: Carrier } \\
\text { Model: } 38 \text { VTA } 040 \\
\text {..90125 Phase-3 } \\
\text { Ins. Date: } 5-5-2014\end{array}$ & $2 \mathrm{~cm}$ & $*$ & $10 \mathrm{mG}$ & $1 \mathrm{~cm}$ & $*$ & $11 \mathrm{mG}$ & $3 \mathrm{~cm}$ & $*$ & $9 \mathrm{mG}$ & $11 \mathrm{mG}$ \\
\hline 3 & $\begin{array}{l}\text { Power: } 3 \text { ton } \\
\text { AC: Carrier, } 2 \mathrm{pcs} \\
3 \text { phase } 50 \mathrm{~Hz} \\
\text { No. } \\
\text { 38VTA040.9015 } \\
\text { Ins. Date: } 5.5 .2014\end{array}$ & $12.2 \mathrm{~cm}$ & $*$ & $13 \mathrm{mG}$ & $1 \mathrm{~cm}$ & $*$ & $13 \mathrm{mG}$ & $2 \mathrm{~cm}$ & $*$ & $14 \mathrm{mG}$ & $14 \mathrm{mG}$ \\
\hline $4 \mathrm{a}$ & $\begin{array}{l}\text { Power- } 3 \text { ton } \\
\text { Brand: Carrier } \\
\text { Model: } 38 \text { VTA } 040 \\
90125 \text { Phase-3, } 50 \\
\text { Hz } \\
\text { Ins. Date: } 5-5-2014\end{array}$ & $43 \mathrm{~cm}$ & $*$ & $10 \mathrm{mG}$ & $21 \mathrm{~cm}$ & $*$ & $17 \mathrm{mG}$ & $2 \mathrm{~cm}$ & $*$ & $10 \mathrm{mG}$ & $17 \mathrm{mG}$ \\
\hline $4 b$ & $\begin{array}{l}\text { Power- } 3 \text { ton } \\
\text { Brand: Carrier } \\
\text { Model: } 38 \text { VTA } 040 \\
90125 \text { Phase-3,50 } \\
\text { Hz } \\
\text { Ins. Date: } 5-5-2014\end{array}$ & $12 \mathrm{~cm}$ & $*$ & $9 \mathrm{mG}$ & $27 \mathrm{~cm}$ & $*$ & $22 \mathrm{mG}$ & $1 \mathrm{~cm}$ & $*$ & $3 \mathrm{mG}$ & $22 \mathrm{mG}$ \\
\hline $4 \mathrm{c}$ & $\begin{array}{l}\text { Power- } 3 \text { ton, AC- } \\
\text { 2pcs } \\
\text { Brand: Carrier } \\
\text { Model: } 38 \text { VTA 040 } \\
\text { 90125 Phase-3, 50 } \\
\text { Hz } \\
\text { Ins. Date: } 5-5-2014\end{array}$ & $2 \mathrm{~cm}$ & $*$ & $6 \mathrm{mG}$ & $8.2 \mathrm{~cm}$ & $*$ & $16 \mathrm{mG}$ & $3.2 \mathrm{~cm}$ & $*$ & $8 \mathrm{mG}$ & $16 \mathrm{mG}$ \\
\hline $4 d$ & $\begin{array}{l}\text { Power-3 ton, AC- } \\
\text { 2pcs } \\
\text { Brand: Carrier } \\
\text { Model: } 38 \text { VTA } 040 \\
90125 \text { Phase-3, } 50 \\
\text { Hz } \\
\text { Ins. Date: } 5-5-2014\end{array}$ & $4.8 \mathrm{~cm}$ & $*$ & $12 \mathrm{mG}$ & $14.5 \mathrm{~cm}$ & $*$ & $25 \mathrm{mG}$ & $4.3 \mathrm{~cm}$ & $*$ & $5 \mathrm{mG}$ & $25 \mathrm{mG}$ \\
\hline $5 a$ & $\begin{array}{l}\text { Power-3 ton } \\
\text { Brand: Carrier } \\
\text { Model: } 38 \text { VTA } 040 \\
90125 \text { Phase-3, } 50 \\
\text { Hz } \\
\text { Ins. Date: } 5-5-2014\end{array}$ & $3 \mathrm{~cm}$ & $*$ & $4 \mathrm{mG}$ & $2 \mathrm{~cm}$ & $*$ & $3 \mathrm{mG}$ & $3 \mathrm{~cm}$ & $*$ & $4 \mathrm{mG}$ & $4 \mathrm{mG}$ \\
\hline $5 b$ & $\begin{array}{l}\text { Power-3 ton } \\
\text { Brand: Carrier } \\
\text { Model: } 38 \text { VTA } 040 \\
90125 \text { Phase-3, } 50 \\
\mathrm{~Hz} \\
\text { Ins. Date: } 5-5-2014\end{array}$ & $1 \mathrm{~cm}$ & * & $10 \mathrm{mG}$ & $2 \mathrm{~cm}$ & $*$ & $9 \mathrm{mG}$ & $3 \mathrm{~cm}$ & $*$ & $10 \mathrm{mG}$ & $10 \mathrm{mG}$ \\
\hline
\end{tabular}




\section{IV.ANALYSIS}

In Table 1, experimental data were collected from the following labs: serial nos. 1a-1h from EE Lab, serial nos. 2a-2e from Textile Testing and Quality Control lab, serial nos.3a-3b from Workshop practice lab, and serial nos. 5a5f are from Physics lab. Lab equipments of various labs were measured for their Electric field and Magnetic field threshold distances as well as the magnetic field values for (i) in front of the equipment measured from the centre of the equipment (ii) at right side of the equipment and (iii) at left side of the equipment. Also, the maximum magnetic fields were measured for the equipments of these labs. The "*" sign indicates that the threshold distance was above recommended level and out of range.

In Table 2, experimental data were collected from the following labs: serial nos. 1a-1b from EE Lab, serial no. 2a-2b from Textile Testing and Quality Control lab, serial no. 3 from Workshop practice lab, serial nos. $4 a-4 d$ are from Yarn manufacturing technology lab, and serial nos. $5 a-5 b$ are from Physics lab. Air Conditioners (AC) brand "Carrier"- having 3 tons of various models were measured for their Electric field and Magnetic field threshold distances as well as the magnetic field values for (i) in front of the equipment measured from the centre of the equipment (ii) at right side of the equipment and (iii) at left side of the equipment. Also, the maximum magnetic fields were measured for each $\mathrm{AC}$ of this lab. The "**" sign indicates that the threshold distance was above recommended level and out of range.

In these labs, it has been observed that the magnitude of those measurements for these fields in meter was fluctuating which was confusing. In some cases, it has been observed that (i) there were not sufficient space for the Air Conditioner and lab equipments and so there were some problems to measure the accurate magnetic field and electric field and (ii) The scale was too short to measure the right side of the AC. EMF readings from the distribution board, switch board and socket points, also difficult to measure the value of the MF machine because fluctuation, at different times got different values.

\section{DISCUSSION}

It was found from the results that the magnetic field values are much higher than the threshold level. From equipments the maximum magnetic field obtained was $600 \mathrm{mG}$ and from air conditioners the maximum magnetic field value was $25 \mathrm{mG}$. Air compressors had the maximum magnetic fields $(600 \mathrm{mG})$ and electric balance had the lowest (9 $\mathrm{mG})$. Because of the nature of the wiring both in the ceiling and floor, all the rooms had higher magnetic field than threshold value. Students work on an average of 3-6 hours a day in those labs. We have in mind to include labs from other departments of Southeast University for the study to continue.

There has been an increase of use of the air conditioners in Bangladesh for the last few years in various offices and organizations to increase the working efficiency of the employees. For this reason, the load shedding of electricity has increased tremendously for the last few years due to excessive air conditioner used in offices, organizations and private homes for comfort living.

\section{CONCLUSION}

From the above lab results, it has been found that in most cases the magnetic field has crossed threshold value. The electric field also has a higher threshold value in some of the equipments. Also, the magnetic field maximum exposure was as high as $600 \mathrm{mG}$ in some cases. Wiring must be done according to the building code 2012. As the locations of air conditioners were on the wall near to the roof, therefore because of this height (distance) students they do not possess that much hazards as it should be. It is hoped that this survey will be helpful as a preventive measure for students and employees of Southeast University.

\section{REFERENCES}

[1] N. Wertheimer N and ED. Leeper. Electrical wiring configurations and childhood cancer. American journal of Epidemiology. 1979, vol. 109 , no. 3 , pp. $273-284$

[2] S. Milham. Mortality from leukaemia in workers exposed to electrical and magnetic fields. New English J Med. 1982, vol. 307, pp. 249.

[3] M. H. Repacholi, An Overview of WHO's EMF Project and the Health Effects of EMF Exposure. Proceedings of the International Conference on Non-Ionizing Radiation at UNITEN (ICNIR 2003), Electromagnetic Fields and Our Health. 2003, pp. 1-13

[4] History of air conditioning. US Department of Energy. Website: http://www.energy.gov/articles/history-air-conditioning (accessed on $10-12-2015)$

[5] I. Nordenson, K. H. Mild, G. Andersson, and M. Sandström. Chromosomal aberrations in human amniotic cells after intermittent exposure to fifty hertz magnetic fields. Bioelectromagnetics. 1994, vol. 15 , no. 4 , pp. $293-301$

[6] M. Quamruzzaman, and M. Haque. Epidemiological Survey of People Working in EMF Field Exposed to High Frequency. Proceedings of the Global Engineering, Science and Technology Conference 2012, 28-29 December, Dhaka, Bangladesh

[7] M. Haque and M. Quamruzzaman. Epidemiological survey on effect of EMF emitted by photocopy machines generally used in Dhaka city Bangladesh. IJRET: International Journal of Research in Engineering and Technology. 2015, vol. 4, no. 4, pp. 92-100

8] M. Quamruzzaman and M. Haque. Survey of EMF emitted by Lab equipments in various labs of Southeast University in Bangladesh for possible preventive health hazards. IFRSA International Journal Of Electronics Circuits And Systems. 2015, vol. 4, no.1, pp. 26-32

[9] M. Quamruzzaman and M. Haque, et al. Effects of electromagnetic fields (EMF) near high voltage transmission line: a case study. Bangladesh Journal of Medical Physics. 2014, vol.7, no.1, pp. 6668

[10] M. Quamruzzaman and M. Haque. Non Ionizing Radiation (NIR), its harmful effects especially from Mobile/Cell Phone and Towers. Southeast University Journal of Science and Engineering. 2014 vol. 8 , no.1, pp. $34-39$ 